\title{
Simulation-based education in Canada: will anesthesia lead in the future?
}

\author{
Robert J. Byrick, MD • Viren N. Naik, MD • \\ J. Earl Wynands, MD
}

Published online: 19 February 2009

(C) Canadian Anesthesiologists' Society 2009

It is remarkable that 40 years have elapsed since Denson and Abrahamson first introduced high fidelity simulation (Sim One) to facilitate the teaching of endotracheal intubation and the induction of anesthesia. ${ }^{1}$ The concept of utilizing a simulator for clinical training was far ahead of its time and not immediately embraced by anesthesiologists or other educators. Simulation technology for medical education underwent a revival in the mid-1980s when computer-generated screen based simulation programs made their appearance, ${ }^{2}$ focusing primarily on pharmacology and physiology applications relevant to anesthesia. The anesthesiologist could choose one of many scenarios that might be encountered by a clinical anesthesiologist. These programs allowed the learner to interpret information and make pharmacological and therapeutic decisions. Such simulators were novel and afforded a meaningful learning experience, especially for novice learners; however, they did not duplicate the application of practical skills and knowledge in a clinical environment in real time.

In 1986, Gaba et al. $^{3}$ at Stanford University developed a full-scale, high fidelity simulator ("The Comprehensive Anesthesia Simulation Environment [CASE]") which allowed the anesthesiologist to manage critical situations. With Jeff Cooper in Boston, they developed an organized program called "Anesthesia Crisis Resource Management" (ACRM), applying principles from the airline industry where

R. J. Byrick, MD ( $₫)$ · V. N. Naik, MD

Department of Anesthesia, St. Michael's Hospital,

30 Bond Street, Toronto, ON M5B 1W8, Canada

e-mail: robert.byrick@utoronto.ca

J. E. Wynands, MD

Department of Anesthesia, University of Ottawa,

22 Rideau View Terrace, Ottawa, ON K2C 3R9, Canada pilots and airline crew use "Crew Resource Management" programs to elicit human responses in a realistic environment. ${ }^{4}$ The objective of ACRM is to teach participants the importance of non-technical skills such as team working, task management, decision-making and situation awareness, focusing on communication and leadership skills.

In the late 90s, several simulation centres appeared in Canada, led by anesthesiologists, to allow learners to practice difficult and rare scenarios without placing patients at risk. ${ }^{5}$ Today, simulation centres have been developed in over 50 centres across Canada, including many community colleges, in addition to hospitals and universities. In spite of the exponential growth of simulation, many professions and disciplines have been slow to recognize that simulationbased education is more than a teaching tool-it is a novel form of "experiential education", with which non-technical skills required in professional practice can be learned. ${ }^{6}$ Educators know that these are difficult (if not impossible) to teach in the clinical environment.

Most Canadian academic departments of anesthesia have been progressive in integrating simulation into the undergraduate and/or postgraduate curricula. Undergraduate medical students who are given time in an anesthesia simulator, enthusiastically compete with each other to manage the airway, and pharmacologically treat simple hemodynamic problems. It is often their first opportunity to independently treat a "patient problem" and the majority find it very enjoyable and a great learning experience. At the postgraduate level, simulators are used to teach firstyear residents how to "troubleshoot" the anesthesia gas machine through clinically relevant scenarios, and manage common intraoperative anesthetic problems. Postgraduate years $2-5$ often spend at least two, three-hour sessions each year in the simulation center. They usually share this experience in a small group and are usually not in the same 
year of training. When they are not in the "hot seat" managing or assisting with the simulated crisis, they are asked to participate in the scenarios, role-playing colleagues from other allied health professions. By playing other roles, residents sometimes achieve a better understanding of the responsibilities and needs of our perioperative colleagues, which can facilitate an appreciation of the importance of non-technical skills. Scenarios are designed to give residents experience with common and uncommon anesthesia problems, while eliciting crisis resource management principles. ${ }^{7}$ A typical scenario lasts about ten to $20 \mathrm{~min}$ and is followed by the critical debriefing session which focuses on both technical and non-technical aspects. Well-trained instructors are never confrontational and negative criticism is avoided to decrease stress and makes for a meaningful reflective learning experience. Each resident signs a confidentiality agreement and negative observations of a resident's performance are never presented to the residency program committee, ensuring a safe learning environment.

Whereas medical students and postgraduate anesthesia residents have shown considerable enthusiasm for simulation training, practicing anesthesiologists have not embraced this learning opportunity. Reasons for this attitude have been reported widely. Most commonly, we have heard "a lack of free/protected time" and/or "the financial consequences of missing work". A majority also state there are no incentives or consequences that hinge on training in a simulatorunlike the airline industry where corporate rules make it mandatory. Given that postgraduate trainees clearly value simulation, it was our hope that these same graduates would use it as an instrument to maintain competency when they are practicing anesthesiologists. Unfortunately, we are now ten years after the first residents graduated with simulation as part of their curriculum, and there is no evidence that this has happened. There is still no centre in Canada where anesthesiologists have voluntarily agreed to undergo regular learning in a high-fidelity simulation environment for continuing professional development.

This prompts us to ask the question: what are anesthesiologists doing to validate their competency as medical practitioners, because it is clear that the public supports and expects regular upgrading of physicians in the practice of their specialty. Levinson has recently published a provocative editorial addressing this problem. ${ }^{9}$ She reminds us that in 2007, a position paper was published by the Federation of Medical Regulatory Authorities of Canada (FMRAC) that proposed that all licensed physicians in Canada must participate in a revalidation process to demonstrate their continued competency within a framework that is fair and relevant. ${ }^{10}$ The Royal College's Maintenance of Certification Program falls short of what is required in the United States, where their programs to maintain certification are more demanding and challenging. Specifically, all specialists in the United States must recertify every ten years. Components of their process which are absent in Canada include submission of a quality improvement practice plan, and a computerized multiple choice examination that tests cognitive skills. Levinson ${ }^{9}$ suggested that there is a need to explore the most effective methods of determining physician maintenance of skills and knowledge. The real question is whether we, as a specialty, can be proactive to adopt innovative, relevant, learning mechanisms, like simulation, voluntarily as part of our "revalidation program" and lead in Continuing Professional Development (CPD). The alternative is to be reactive. Will it be necessary for hospitals and professional colleges, through the FMRAC, to enforce such programs, potentially through high stakes "pass/fail" examinations? If we become proactive, we will be empowered to establish best practice in CPD, and guide the content and accreditation of our own revalidation programs. Certainly, this process has started in the United States where there is a concerted effort in many quarters (e.g., American College of Surgeons, Society for Simulation in Healthcare, American Society of Anesthesiologists [ASA]) to accredit Simulation Centres and programs. The purpose of the ASA Committee on Simulation's program is to establish a network of endorsed anesthesia simulation programs, which meet strict criteria, and expand CPD using simulation for its members. ${ }^{11}$ To date, this has not been done in Canada by the Canadian Anesthesiologists' Society. The "Canadian Network for Simulation in Healthcare" has been formed by the Canadian Patient Safety Institute (CPSI). CPSI believes simulation technology provides a method to learn and evaluate non-technical skills as critical elements of medical practice. Concurrently, the Royal College has created a task force to address simulation needs in Canada and work synergistically with CPSI. If site accreditation falls under the mandate of either of these organizations, anesthesiologists need to be involved.

Validation of the effectiveness of anesthesia training using a high-fidelity simulator is another question. Wong ${ }^{12}$ examined teaching, training, evaluation and research in high-fidelity simulation. She concluded that the costeffectiveness, efficacy compared to traditional training and the impact on patient outcomes is still pending. She emphasized that medical education has changed from a process and structure-based curriculum, to an outcome or competency based curriculum. In the former, learning is based on an exposure to a defined curriculum over a specified time, while the latter is based on the attainment of preset objectives and competencies. An obvious problem is the defining of objectives and establishing competency criteria. Simulation may provide an instrument to aid the transition to competency based assessment. The Royal 
College has recently become so convinced of simulation's potential for competency based assessment, that it will be slowly incorporated into the examination process in various disciplines. There is little doubt that this advance will lead to more widespread curricular integration, as we know that evaluation often drives curriculum.

$\mathrm{Gaba}^{13}$ emphasized that no industry which depends on the rapid response of skilled individuals within a system for life-threatening events has waited for unequivocal proof of the benefits before implementing simulation-based education. While we wait for the establishment of standards, guidelines, and accreditation for simulation-based education for CPD and revalidation, the use of the best available evidence and techniques should proceed. Ziv et al. ${ }^{14}$ have argued persuasively that there is an "ethical imperative" to use simulation as it conveys to patients, learners, educators and society that patients are to be protected whenever possible and are not conveniences for our training program. If our specialty in Canada moves forward with this agenda, we have a unique opportunity to evaluate the role of simulation in all phases of our educational programs. In addition, the public values professionals who use simulation in other industries where innocent people are placed at risk and they wonder how any physician could say "no" to practice. Will the profession meet the challenge, showing leadership? This could be accomplished by using simulation voluntarily to enhance patient safety. This could be a powerful public relations opportunity for our national society and for the profession of anesthesiology in Canada. Will anesthesiologists lead again?

\section{La formation à l'aide de simulateurs au Canada: l'anesthésie sera-t-elle le fer de lance à l'avenir ?}

Il est tout à fait remarquable que 40 ans se soient écoulés depuis que Denson et Abrahamson aient introduit le premier simulateur haute fidélité (Sim One) afin de faciliter l'enseignement de l'intubation endotrachéale et l'induction de l'anesthésie. ${ }^{1}$ Le concept-même d'utiliser un simulateur pour la formation clinique était avant-gardiste et n'a pas été adopté immédiatement par les anesthésiologistes et autres responsables de l'enseignement. La technologie de simulation en formation médicale a connu une renaissance au milieu des années 1980 lorsque sont apparus des programmes de simulation basés sur des écrans d'ordinateurs. ${ }^{2}$ Ces programmes traitaient principalement des applications pharmacologiques et physiologiques pertinentes à l'anesthésie. L'anesthésiologiste pouvait choisir un scénario parmi plusieurs qui pouvaient potentiellement survenir dans la pratique clinique. Ces programmes permettaient à l'étudiant d'interpréter des informations et de prendre des décisions pharmacologiques et thérapeutiques. Ces simulateurs étaient novateurs et ont procuré une expérience d'apprentissage significative et ce, particulièrement pour les novices; toutefois, ils ne reproduisaient pas l'application de compétences pratiques et des connaissances dans un environnement clinique en temps réel.

En 1986, Gaba et coll. ${ }^{3}$ ont mis au point un simulateur haute fidélité grandeur nature à l'Université Stanford ("The Comprehensive Anesthesia Simulation Environment [CASE]") qui donnait l'occasion à l'anesthésiologiste de prendre en charge des situations critiques. Avec la collaboration de Jeff Cooper à Boston, ils ont élaboré un programme de gestion des crises baptisé «Anesthesia Crisis Resource Management »(ACRM), en appliquant des principes utilisés dans l'aviation, où les pilotes et l'équipage utilisent des programmes de gestion des capacités de l'équipage ( «Crew Resource Management») pour susciter des réactions humaines dans un environnement réaliste. ${ }^{4}$ L'objectif de l'ACRM est d'inculquer l'importance de compétences non techniques telles que le travail en équipe, la gestion des tâches, la prise de décision et la conscientisation de la situation aux participants, avec une emphase sur les compétences de communication et de leadership.

À la fin des années 90, plusieurs centres de simulation dirigés par des anesthésiologistes ont vu le jour au Canada afin de permettre aux étudiants d'acquérir des compétences pratiques et de les exercer dans des situations difficiles et rares sans mettre les patients en danger. ${ }^{5}$ Aujourd'hui, il existe des centres de simulations dans plus de 50 centres au Canada, notamment dans plusieurs collèges communautaires, outre les hôpitaux et universités. Malgré la croissance exponentielle de la simulation, nombreuses sont les professions et les disciplines qui tardent à reconnaître que la formation en simulateur constitue plus qu'un simple outil d'enseignement, mais véritablement une nouvelle forme de formation 'expérientielle' grâce à laquelle les compétences non techniques nécessaires à la pratique professionnelle peuvent être apprises. ${ }^{6}$ Les responsables de l'éducation sont conscients que ces compétences sont difficiles, voire impossibles, à enseigner dans un environnement clinique.

La plupart des départements universitaires d'anesthésie au Canada ont progressivement intégré la simulation dans leurs programmes de cours de premier et/ou de deuxième et troisième cycle. Les étudiants de premier cycle en médecine qui ont l'occasion de travailler avec un simulateur d'anesthésie rivalisent d'entrain pour prendre en charge les voies aériennes et traiter les problèmes hémodynamiques simples à l'aide d'outils pharmacologiques. Souvent, il s'agit de leur première chance de traiter un 'problème 
présenté par le patient'; la majorité des étudiants apprécient beaucoup cette occasion et la considèrent comme une excellente expérience d'apprentissage. Au niveau gradué, les simulateurs sont utilisés pour enseigner aux résidents de $1^{\text {ère }}$ année comment 'réparer' l'appareil d'anesthésie par inhalation par l'intermédiaire de scénarios pertinents d'un point de vue clinique, et prendre en charge les problèmes peropératoires courants dans la pratique anesthésique. Entre la deuxième et la cinquième année, les résidents passent habituellement au moins deux sessions de trois heures par année dans un centre de simulation. En général, cette expérience se fait dans un petit groupe composé d'étudiants de différentes années. Lorsqu'ils ne sont pas 'aux commandes', gérant ou aidant à gérer la crise simulée, les étudiants prennent part aux scénarios en jouant le rôle de collègues d'autres professions associées de la santé. En jouant ces rôles, les étudiants acquièrent une meilleure compréhension des responsabilités et des besoins des collègues avec lesquels nous travaillons dans le contexte périopératoire, ce qui facilite une appréciation de l'importance des compétences non techniques. Les scénarios sont élaborés de manière à donner aux résidents de l'expérience face aux problèmes courants et moins courants en anesthésie tout en mettant en œuvre les principes de gestion des crises. ${ }^{7}$ Un scénario typique dure de 10 à 20 minutes environ et est suivi par une séance de verbalisation critique qui porte aussi bien sur les aspects techniques que non techniques. Les instructeurs bien formés évitent les confrontations ouvertes et les critiques négatives afin de réduire le stress et créer une expérience d'apprentissage réfléchie et significative. Chaque résident signe un accord de confidentialité et les observations négatives quant à la performance d'un résident ne sont jamais transmises au comité du programme de résidence, ce qui garantit un environnement d'apprentissage sécuritaire.

Alors que les étudiants en médecine et les résidents en anesthésie se sont montrés très enthousiastes envers la formation sur simulateur, les anesthésiologistes praticiens n'ont pas saisi cette occasion d'apprentissage. Plusieurs raisons de cette attitude ont été données. La plus courante de ces raisons est « un manque de temps libre/protégé » et/ou « le manque à gagner lorsqu'ils ne sont pas au travail $»^{8}$ Une majorité déclare également qu'il n’y a pas d'incitatifs ou de conséquences qui s'appuient sur la formation en simulateur - au contraire de la situation dans le secteur de l'aviation, où ce type de formation est rendu obligatoire par les règlements professionnels. Au vu de la valeur que les gradués accordent à la formation sur simulateur, nous espérions que ces mêmes étudiants, une fois devenus des anesthésiologistes praticiens, continueraient d'utiliser cette méthode pour maintenir leurs compétences. Malheureusement, cela fait maintenant dix ans que les premiers résidents ayant bénéficié de la formation en simulateur pratiquent l'anesthésie, et nous n'avons pas encore vu cela arriver. Il n'existe toujours pas de centre au Canada où les anesthésistes ont consenti, sur une base volontaire, à entreprendre un apprentissage régulier dans un environnement de simulation haute fidélité à des fins de formation professionnelle continue.

Dès lors, la question suivante se pose : que font les anesthésiologistes pour valider leurs compétences en tant que praticiens ? En effet, il est évident que le public appuie le principe d'une mise à jour régulière des connaissances et compétences des médecins dans la pratique de leur spécialité et s'attend à des actions en ce sens. Levinson a récemment signé un éditorial provocateur traitant de ce problème. ${ }^{9}$ Elle nous rappelle qu'en 2007, la Fédération des ordres des médecins du Canada (FMRAC) a publié un article de principe suggérant que tous les médecins diplômés au Canada doivent prendre part à un processus de revalidation afin de démontrer que leurs compétences sont à jour dans un cadre juste et pertinent. ${ }^{10}$ Le programme de maintien du certificat du Collège royal ne correspond pas aux exigences américaines, où les programmes de maintien du certificat sont plus exigeants et stimulants. En particulier, aux États-Unis tous les spécialistes doivent repasser une certification tous les dix ans. Parmi les éléments de ce processus qui font défaut aux programmes canadiens, citons la soumission d'un plan pratique d'amélioration de la qualité et un examen à choix de réponses informatisé pour tester les aptitudes cognitives. Dans son éditorial, Levinson ${ }^{9}$ avance qu'il existe un besoin d'explorer les méthodes les plus efficaces pour déterminer le maintien des aptitudes et des connaissances des médecins. La question est de savoir si nous, anesthésiologistes, en tant que spécialité, saurons être proactifs dans notre adoption de méthodes d'apprentissage innovantes et pertinentes, comme la simulation - et ce, de façon volontaire dans le cadre de notre " programme de revalidation » - afin d'être le fer de lance du développement professionnel continu (DPC). L'alternative : être réactif. Sera-t-il nécessaire qu'hôpitaux et collèges professionnels, par l'intermédiaire de la FMRAC, imposent de tels programmes, avec à la clé, peut-être, des examens à enjeu élevé (passage/échec) ? En revanche, si nous devenons proactifs, nous aurons la possibilité de mettre sur pied les meilleures pratiques en DPC et d'orienter le contenu et la certification de nos propres programmes de revalidation. Ce processus est né aux EtatsUnis, où l'on observe un effort collectif provenant de plusieurs directions (par ex. l'American College of Surgeons, Society for Simulation in Healthcare, American Society of Anesthesiologists [ASA]) pour certifier les centres et les programmes de simulation. L'objectif du programme du Comité de l'ASA sur la simulation est de créer un réseau de programmes approuvés de simulation en anesthésie, programmes qui répondraient à des critères 
stricts, et d'étendre le DPC utilisant la simulation à tous ses membres. ${ }^{11}$ À ce jour, la Société canadienne des anesthésiologistes n'a pas mis en place un tel réseau au Canada. L'Institut canadien pour la sécurité des patients (ICSP) a créé le « Réseau canadien pour la simulation dans les soins de santé ». L'ICSP estime que la technologie de simulation offre une méthode d'apprentissage et d'évaluation des compétences non techniques en tant qu'éléments cruciaux de la pratique médicale. Dans le même temps, le Collège royal a créé un groupe d'étude pour répondre aux besoins en matière de simulation au Canada et travailler de concert avec l'ICSP. Si la certification des centres de simulation tombe sous la responsabilité d'un de ces organismes, l'implication des anesthésiologistes sera nécessaire.

La validation de l'efficacité des programmes de formation en anesthésie utilisant un simulateur haute fidélité constitue une autre question. Wong ${ }^{12}$ a passé en revue l'enseignement, la formation, l'évaluation et la recherche touchant à l'utilisation d'un simulateur haute fidélité. Selon ses conclusions, la rentabilité, l'efficacité comparativement aux méthodes de formation classiques, et l'impact sur les devenirs des patients restent encore à être démontrés. Elle souligne que la formation médicale s'est transformée d'un programme d'enseignement basé sur les processus et la structure en un programme basé sur les devenirs ou les compétences. Dans le premier modèle, l'apprentissage se fonde sur l'exposition des étudiants à un programme d'enseignement défini sur une période donnée, alors que le second s'appuie sur l'atteinte d'objectifs et de compétences prédéterminés. Un problème évident se pose dans la définition des objectifs et la mise en place de critères de compétence. La simulation pourrait devenir un instrument qui faciliterait cette transition vers une évaluation basée sur les compétences. Le Collège royal est récemment devenu convaincu du potentiel de la simulation pour l'évaluation basée sur les compétences, à tel point que la simulation sera progressivement intégrée dans le processus d'examen de plusieurs disciplines. Il ne fait aucun doute que cette évolution incitera une intégration plus répandue de la simulation dans les programmes d'enseignement (nous savons tous que l'évaluation est souvent un moteur de changement des programmes d'enseignement).

$\mathrm{Gaba}^{13}$ souligne qu'aucune industrie dépendant de la réaction rapide d'individus compétents dans un système d'événements potentiellement fatals n'a attendu de preuves irréfutables quant aux avantages de la simulation avant de mettre en place une formation basée sur simulateur. Pendant que nous attendons que des normes, des directives et un programme de certification soient mis en place pour la formation basée sur simulation en DPC et la revalidation de nos compétences, nous devons faire avancer l'utilisation des meilleures données probantes et techniques disponibles. Ziv et coll. ${ }^{14}$ soutiennent de façon convaincante qu'il existe un « impératif déontologique » en faveur de l'utilisation de la simulation, étant donné que cette méthode d'apprentissage transmet aux patients, étudiants, responsables de l'enseignement et à la société en général que les patients doivent être protégés autant que possible et ne sont pas des cobayes de nos programmes de formation. Si notre spécialité au Canada parvient à évoluer avec cet agenda, nous aurons une occasion unique d'évaluer le rôle de la simulation à tous les stades de nos programmes de formation. De plus, le public tient en haute estime les professionnels qui se servent de la simulation dans d'autres secteurs dans lesquels des innocents sont potentiellement en danger - et se demandent comment un médecin pourrait dire «non » à cette pratique. Notre profession relèvera-t-elle le défi, et montrera-t-elle son leadership ? Pour ce faire, nous pourrions utiliser la simulation sur une base volontaire afin d'améliorer la sécurité des patients. Cela pourrait également constituer une excellente occasion de relations publiques pour notre société nationale et pour la profession de l'anesthésiologie au Canada ? Les anesthésiologistes seront-ils à nouveau au sommet ?

Conflicts of interest None declared.

Related interests Dr. Wynands is the former Acting Director of the Multidisciplinary Simulation Centre of the University of Ottawa.

\section{References}

1. Denson JS, Abrahamson S. A computer-controlled patient simulator. JAMA 1969; 208: 504-8.

2. Schwid HA. A flight simulator for general anesthesia training. Comput Biomed Res 1987; 20: 64-75.

3. Gaba DM, DeAnda A. A comprehensive anesthesia simulation environment: re-creating the operating room for research and training. Anesthesiology 1988; 69: 387-94.

4. Holzman R, Cooper JB, Gaba DM, Phillip JH, Small SD, Feinstein $D$. Anesthesia crisis resource management: real-life simulation training in operating room crisis. J Clin Anesth 1995; 7: 675-87.

5. Kurrek MM, Fish KJ. Anaesthesia crisis resource management training: an intimidating concept, a rewarding experience. Can J Anaesth 1996; 43: 430-4.

6. Fletcher GC, McGeorge P, Flin RH, Glavin RJ, Maran NJ. The role of non-technical skills in anaesthesia: a review of current literature. Br J Anaesth 2002; 88: 418-29.

7. Gaba DM, Fish KJ, Howard SK. Principles of crisis resource management. In: Gaba DM, Fish KJ, Howard SK, editors. Crisis Resource Management in Anesthesiology. New York: Churchill Livingstone Inc.; 1994. p. 31-52.

8. Savoldelli G, Naik VN, Hamstra SJ, Morgan PJ. Barriers to use of simulation based education. Can J Anesth 2005; 52: 944-50.

9. Levinson $W$. Revalidation of physicians in Canada: are we passing the test? CMAJ 2008; 179: 979-80.

10. Federation of Medical Regulatory Authorities of Canada Revalidation Working Group. Physician revalidation: maintaining competence and performance. Ottawa, ON: The Federation; 2007. 
www.fmrac.ca/committees/documents/final_reval_position_eng. pdf. Accessed 1 Jan 2009.

11. Steadman RH. ASA endorsement of simulation programs under way: committee on simulation education, ABA support innovative CME for ASA members. Am Soc Anesthesiol Newsl 2008; 72: 25-6.
12. Wong $A K$. Full scale computer simulators in anesthesia training and evaluation. Can J Anesth 2004; 51: 455-64.

13. Gaba DM. Improving anesthesiologists' performance by simulating reality. Anesthesiology 1992; 76: 491-4.

14. Ziv A, Wolpe PR, Small S, Glick S. Simulation-based medical education: an ethical imperative. Acad Med 2003; 78: 783-8. 